\title{
Contrasting the Antarctic and Arctic Atmospheric Responses to Projected Sea Ice Loss in the Late Twenty-First Century
}

\author{
MARK ENGLAND \\ Applied Physics and Applied Mathematics Department, Columbia University, New York, New York \\ LORENZO POLVANI \\ Applied Physics and Applied Mathematics Department, and Department of Earth and Environmental Science, \\ Lamont-Doherty Earth Observatory, Columbia University, New York, New York \\ LANTAO SUN \\ Cooperative Institute for Research in Environmental Sciences, University of Colorado Boulder, and \\ NOAA/Earth System Research Laboratory, Boulder, Colorado
}

(Manuscript received 4 October 2017, in final form 7 May 2018)

\begin{abstract}
Models project that Antarctic sea ice area will decline considerably by the end of this century, but the consequences remain largely unexplored. Here, the atmospheric response to future sea ice loss in the Antarctic is investigated, and contrasted to the Arctic case, using the Community Earth Systems Model (CESM) Whole Atmosphere Coupled Climate Model (WACCM). Time-slice model runs with historic sea ice concentrations are compared to runs with future concentrations, from the late twenty-first century, in each hemisphere separately. As for the Arctic, results indicate that Antarctic sea ice loss will act to shift the tropospheric jet equatorward, an internal negative feedback to the poleward shift associated with increased greenhouse gases. Also, the tropospheric response to Antarctic sea ice loss is found to be somewhat weaker, more vertically confined, and less seasonally varying than in the case of Arctic sea ice loss. The stratospheric response to Antarctic sea ice loss is relatively weak compared to the Arctic case, although it is here demonstrated that the latter is still small relative to internal variability. In contrast to the Arctic case, the response of the ozone layer is found to be positive (up to 5 Dobson units): interestingly, it is present in all seasons except austral spring. Finally, while the response of surface temperature and precipitation is limited to the southern high latitudes, it is nonetheless unable to impact the interior of the Antarctic continent, suggesting a minor role of sea ice loss on recent Antarctic temperature trends.
\end{abstract}

\section{Introduction}

Although Antarctic sea ice extent has been increasing steadily at a small but significant rate of nearly $2 \%$ decade $^{-1}$ over the last 40 years (Comiso et al. 2017), it is hard to imagine, under the current trajectory of greenhouse gas emissions, a scenario without a significant reduction in sea ice extent by the end of this century. In fact, 2017 and 2018 were at, or near, record lows for Antarctic March sea ice extent (NSIDC 2018). In all of the representative concentration pathways (RCPs) examined by the Intergovernmental Panel on Climate Change's Fifth Assessment Report, both Arctic and

Corresponding author: Mark England,mre2126@columbia.edu
Antarctic sea ice extent are projected to decrease by 2100 (Collins et al. 2013). The atmospheric response to future Antarctic sea ice loss under a changing climate is, at present, a largely unexplored question.

The recent literature has almost entirely focused on the atmospheric effects of Arctic sea ice loss [for recent reviews see e.g., Cohen et al. (2014) and Vihma (2014)], partly motivated by the recent dramatic reduction of Arctic sea ice extent at a rate of over $4 \%$ decade $^{-1}$ (Vaughan et al. 2013). The consequences of diminishing Arctic sea ice on the high latitudes are well established, with higher levels of warming in the Arctic than at lower latitudes, and increased precipitation in that region (Deser et al. 2010; Screen and Simmonds 2010). There is less agreement, however, on the impact of Arctic sea ice 
loss on the midlatitudes (Barnes and Screen 2015; Overland et al. 2015; Screen 2017a). Evidence from climate models suggests that Arctic sea ice loss acts to shift the jet equatorward in winter (Butler et al. 2010; Deser et al. 2010; Peings and Magnusdottir 2014; Sun et al. 2015, hereafter S15) and the robustness among models has been documented in Screen et al. (2018). However, many features of the midlatitude response to sea ice loss identified from the recent historical trends (Screen et al. 2013; Mori et al. 2014; McCusker et al. 2016; Sun et al. 2016) seem to be model dependent.

For instance, there is disagreement over whether sea ice loss causes a weakening (Kim et al. 2014; Peings and Magnusdottir 2014; S15) or a strengthening (Cai et al. 2012; Screen et al. 2013; Sun et al. 2014) of the polar vortex. S15 demonstrate that part of the discrepancy can be reconciled by the competing effect from the sea ice loss in the Pacific and Atlantic sector: melting sea ice in the Atlantic sector is linked to enhanced wave propagation into the stratosphere and a weaker vortex whereas melting sea ice in the Pacific sector is linked to wave suppression and a stronger vortex. This cancellation, also reported in McKenna et al. (2018), is found to produce a muted stratospheric response when sea ice loss is imposed in both sectors.

Unlike the Arctic, there has been very little research concentrating on the atmospheric impacts of projected Antarctic sea ice loss. The Antarctic offers the opportunity to investigate the effects of future sea ice loss in a simpler setting than the Arctic because 1) most of the major landmasses are found in the Northern Hemisphere, 2) the Southern Hemisphere atmospheric circulation is more zonally symmetric, and 3 ) projected sea ice losses are much less regionally varying in the Antarctic. In the context of historical trends, it has been suggested that recent sea ice expansion might have resulted in a small poleward jet shift during the cold seasons (Smith et al. 2017). Results from Raphael et al. (2011) suggest that changes in Antarctic sea ice in austral summer may also have an effect on the atmospheric circulation. Complicating matters, Bracegirdle et al. (2018) suggest that the relationship between Antarctic sea ice extent and the strength of the southern jet is possibly stronger than the relationship with the latitudinal position of the jet. On the subject of future Antarctic sea ice decline, we are aware of only three studies that have used comprehensive climate models, and their results are in disagreement; Kidston et al. (2011) find no significant effects when contracting the sea ice edge, whereas Bader et al. (2013) (while investigating the response in austral winter alone) find an equatorward shift of the tropospheric jet, in agreement with an earlier study by Menendez et al. (1999). The effect of the changing Antarctic sea ice on the midlatitudes is still largely an unresolved question.

In this paper, we examine the atmospheric response to future reduced Antarctic sea ice extent, and contrast it with its Arctic counterpart, using a state-of-the-art climate model. Following S15, we use the National Center for Atmospheric Research (NCAR) Whole Atmosphere Community Climate Model (WACCM), a model with a well-resolved stratosphere and interactive chemistry for stratospheric ozone. We find that projected Antarctic sea ice losses have a clear and robust effect on the tropospheric circulation throughout the year, albeit with an amplitude that is somewhat smaller compared to the Arctic response.

This paper is organized as follows. In Section 2, we describe the model and experimental design used in this study. In section 3, we compare and contrast the atmospheric response, in the troposphere, stratosphere, and at the surface, to future Antarctic and Arctic sea ice loss. The paper is concluded with a summary and discussion in section 4 .

\section{Data and methods}

\section{a. Model}

For this study we employ the WACCM4, a high top model that participated in phase 5 of the Coupled Model Intercomparison Project (CMIP5), and was fully documented by Marsh et al. (2013). It has a horizontal resolution of $1.9^{\circ}$ latitude by $2.5^{\circ}$ longitude, with 66 vertical levels and a model lid extending up to the lower thermosphere, near $140 \mathrm{~km}$. In addition to enhanced vertical resolution in the stratosphere and mesosphere, WACCM incorporates an interactive stratospheric chemistry package, and special gravity wave parameterizations for the upper atmosphere. These features give this model a much improved representation of the stratosphere than low top models. Employing a high top model is important because recent research has identified the stratosphere as a possible pathway for interactions between the polar climate and midlatitude weather (Peings and Magnusdottir 2014; Zhang et al. 2018).

\section{b. Experiments}

We investigate the effect of Antarctic and Arctic sea ice loss on the atmospheric circulation by performing three numerical experiments, each 151 years long, using WACCM: a control run and two perturbed sea ice runs. The control experiment is forced with a repeating seasonal cycle of sea ice concentration (SIC) and sea surface temperature (SST), averaged over the period 
1955-69 (the reason for this choice is discussed below), obtained from the mean of a six-member ensemble of historical twentieth-century simulations with the corresponding fully coupled version of WACCM (Marsh et al. 2013). The two perturbation experiments are specified with repeating seasonal cycles of polar SIC in one hemisphere, averaged over the period 2085-99, from the mean of a six-member ensemble of twentyfirst-century simulations of the fully coupled version of WACCM, forced by RCP8.5. The perturbed Antarctic experiment has prescribed 2085-99 values for Antarctic SIC, but Arctic SIC is unchanged from the control. Similarly, the perturbed Arctic experiment has prescribed 2085-99 values for Arctic SIC, but Antarctic SIC is left unchanged from the control. The SSTs in the perturbation experiments are handled using the same method as in S15: in the perturbation experiments, SSTs are changed to end of the century values, averaged from the six WACCM RCP8.5 runs, only in grid cells where SIC is projected to decrease. Following convention (e.g., Peings and Magnusdottir 2014), sea ice thickness is specified to be $2 \mathrm{~m}$ in the Arctic and $1 \mathrm{~m}$ in the Antarctic.

While it is common to use the year 2000 (or present conditions) for the control runs, in our experiments all forcings (including ozone-depleting substances) are fixed at 1955 year levels, except for SIC. In all other aspects, the design of these experiments is similar to the Arctic runs performed by S15. However, we have chosen to use an earlier period for the control because we are primarily focusing on the Southern Hemisphere. By fixing all forcings at 1955 values, we can avoid including a perpetual stratospheric ozone hole in the simulations [recall that the Antarctic polar stratosphere is severely perturbed at present, but the ozone hole is expected to recover by 2060-80; WMO (2014)]. To avoid complicating matters with the recovery of stratospheric ozone, we have selected the SIC from the middle of the twentieth century, before the ozone hole over Antarctica was ever formed. This choice results in a slightly larger amount of sea ice loss than the one imposed by S15; however, since the vast majority of the loss is projected to occur in the twenty-first century, the results of these two studies are broadly comparable in the Arctic.

\section{c. The imposed sea ice forcing}

Given our experiment design, it should be clear that differences found in the atmospheric circulation between the Antarctic perturbation experiment and the control experiment can be attributed to Antarctic sea ice loss, although a small remnant of internal variability might be present, even with such long integrations (150 years). Similarly, differences found in atmospheric circulation between the Arctic perturbation experiment and the control experiment can be attributed to Arctic sea ice loss. Therefore, we start by examining the SIC forcing, and contrasting the Antarctic and Arctic losses of sea ice.

Figure 1 shows the differences between the perturbation experiments and the control experiment for the Arctic SIC (first row) and Antarctic SIC (third row). SIC is projected to diminish in all seasons in both hemispheres by the end of the century. In the Northern Hemisphere, significant sea ice loss is found in the Arctic Circle in summer and autumn. In the winter and spring, sea ice loss is restricted to the marginal zones, especially the Bering Sea, Hudson Bay, the Sea of Okhotsk, and the Barents Sea. In comparison, the predicted sea ice loss in the Southern Hemisphere appears far more zonally symmetric. In austral winter and spring the projected sea ice loss is an annulus completely encircling the Antarctic continent, whereas in austral autumn future sea ice loss is confined to the Antarctic coastline. The net heat flux (the sum of the longwave flux, the sensible heat flux, and the latent heat flux) associated with these sea ice changes is also shown (second and fourth row) with, as expected, areas of sea ice melt losing energy to the atmosphere. Equatorward of the sea ice loss region, the atmosphere can be warmed by thermal advection while SSTs are fixed by experimental design. This leads to a downward heat flux response, a common feature in similar modeling studies (e.g., Deser et al. 2010).

The area-integrated quantities of sea ice and net heat flux, and their variations throughout the year, are presented in Fig. 2. The seasonal cycle of sea ice area (SIA) for the control (averaged 1955-69) and the perturbed experiments (averaged 2085-99) are shown for the Arctic (Fig. 2a) and the Antarctic (Fig. 2b). Consistent with the theory of Eisenman et al. (2011), SIA becomes less seasonally varying in the Antarctic by the end of the century, whereas the seasonal cycle of Arctic sea ice becomes larger. This is more easily interpreted by considering the timing of the maximum Arctic sea ice loss (Fig. 2c), which aligns with the Arctic September minimum (Fig. 2a), whereas the minimum Antarctic sea ice loss (Fig. 2c) coincides with the Antarctic February minimum (Fig. 2b).

The annual cycle of net surface flux (Fig. 2d) and total forcing to the atmosphere (Fig. 2e), arising from the SIC changes, are lagged by a few months after the timing of the maximum sea ice loss in the Arctic, but this does not seem to be the case in the Antarctic. Recall that changes in heat flux need not be in phase with changes in sea ice loss; for example, in the Arctic, the energy transport from the ocean to the atmosphere is highest in winter 

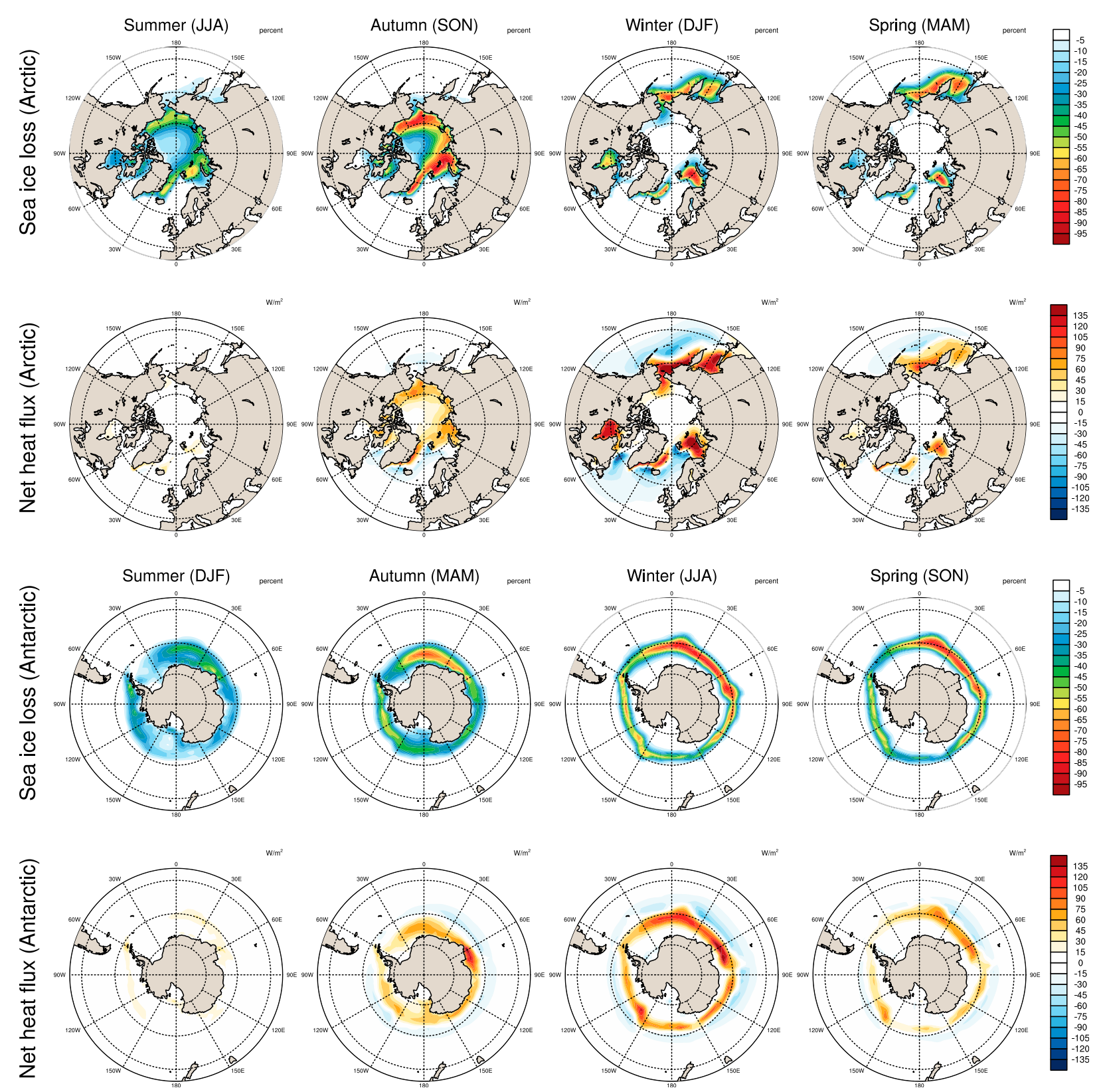

FIG. 1. Seasonally averaged sea ice loss (difference between 1955-69 and 2085-99 averages) imposed for the (first row) Arctic and (third row) Antarctic experiment, and the associated net surface heat fluxes in the (second row) Arctic and (fourth row) Antarctic. Positive heat fluxes correspond to the ocean releasing heat to the atmosphere and negative heat fluxes correspond to the atmosphere losing heat to the ocean. Note that seasons have been shifted to align northern and austral seasons.

even though sea ice loss peaks in the autumn. The details of how the net surface flux and the total forcing to the atmosphere were calculated are included in the appendix. We expect to see the largest atmospheric circulation response to sea ice loss in months where the net surface flux peaks, so winter for the Arctic, and autumn and winter for the Antarctic. In addition to the role played by the net surface fluxes, the basic climatological state of the atmosphere is also important, as shown by Smith et al. (2017).

The experimental setup for the experiments is summarized in Table 1. The following figures will all show the difference (future - past) between the perturbation and the control experiments: we will refer to these as the "response" to sea ice loss. In all of the following figures the months have been aligned so that boreal and austral 


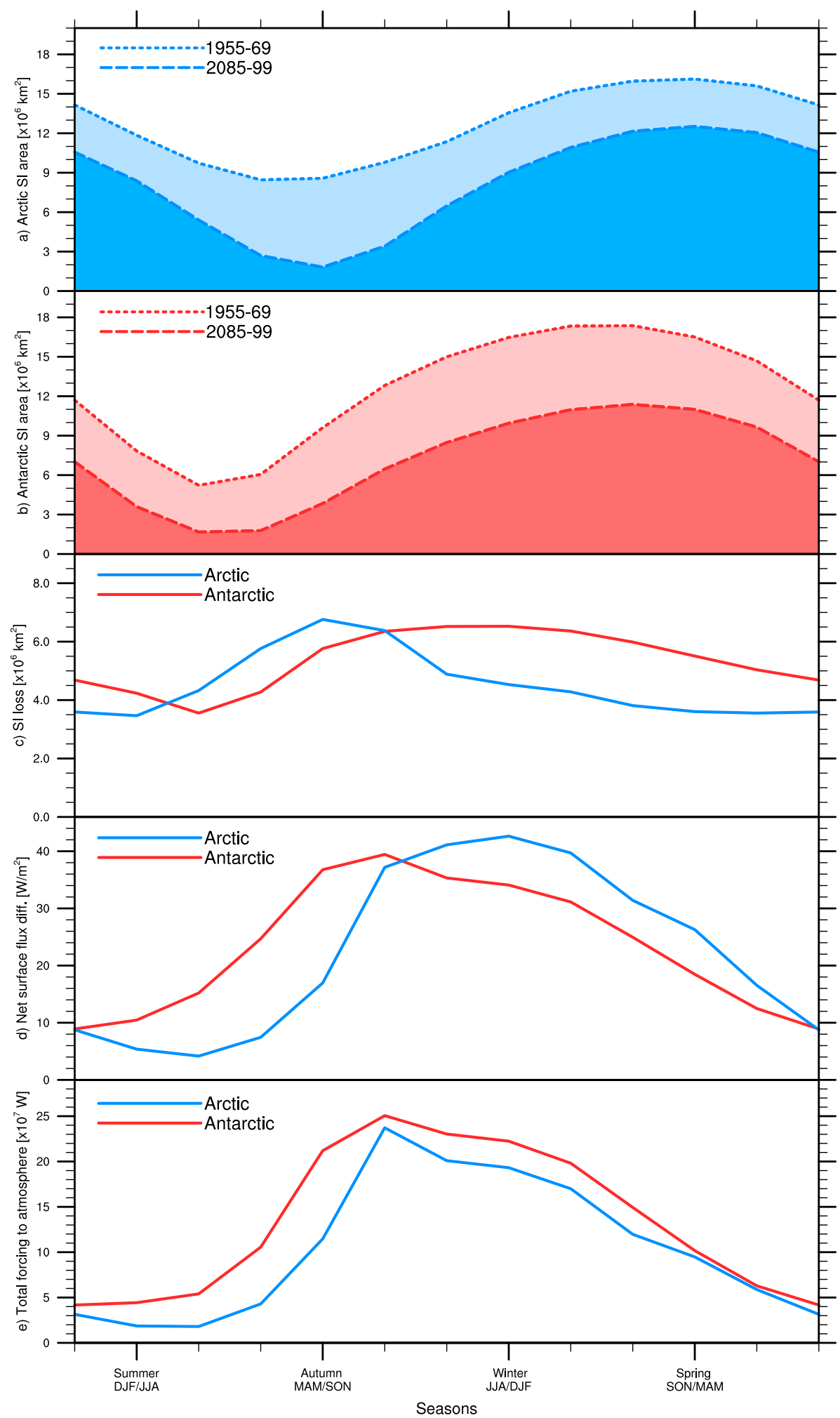

FIG. 2. The seasonal cycle of 1955-69 and 2085-99 total sea ice area for (a) the Arctic and (b) the Antarctic, from historical and RCP8.5 WACCM runs, respectively. (c) For both the Arctic and Antarctic the monthly sea ice loss between these two periods is shown. Resulting from this sea ice loss are the (d) net surface flux differences and (e) the total forcing to the atmosphere [values in (c) multiplied by values in (d)]. Note that the months have been shifted to align northern and austral seasons. 
TABLE 1. Details of the three WACCM time slice experiments and the sea ice conditions imposed in them. Sea ice conditions averaged over the years 1955-69 are from the ensemble mean of six WACCM historical runs. Sea ice conditions averaged over the years 2085-99 are from the ensemble mean of six WACCM RCP8.5 runs.

\begin{tabular}{|c|c|c|c|c|}
\hline Expt & Antarctic sea ice & Arctic sea ice & Years & All other forcings \\
\hline Control (C) & $1955-69$ & $1955-69$ & 151 & Fixed at 1955 values \\
\hline Future Antarctic (A1) & 2085-99 & $1955-69$ & 151 & Fixed at 1955 values \\
\hline Future Arctic (A2) & $1955-69$ & 2085-99 & 151 & Fixed at 1955 values \\
\hline
\end{tabular}

seasons correspond with each other. To calculate the strength and latitudinal position of the tropospheric jet, we use the approach of Bracegirdle et al. (2018). The zonally averaged zonal wind between $75^{\circ}$ and $15^{\circ} \mathrm{S}$ (Southern Hemisphere jet) and $15^{\circ}$ and $75^{\circ} \mathrm{N}$ (Northern Hemisphere jet) is interpolated with a cubic spline onto a $0.1^{\circ}$ latitude grid; from this interpolated curve, the maximum wind speed and its location is determined for each month. A two-sided Student's $t$ test is used to estimate the statistical significance of all responses. We discard the first year of each 151-yr simulation from our analysis.

\section{The atmospheric response to future sea ice loss}

\section{a. Tropospheric response}

Figure 3 shows the zonally averaged temperature response (shading) throughout the whole atmosphere to Arctic (top row) and Antarctic (bottom row) projected

\section{Temperature changes due to Arctic SI loss}
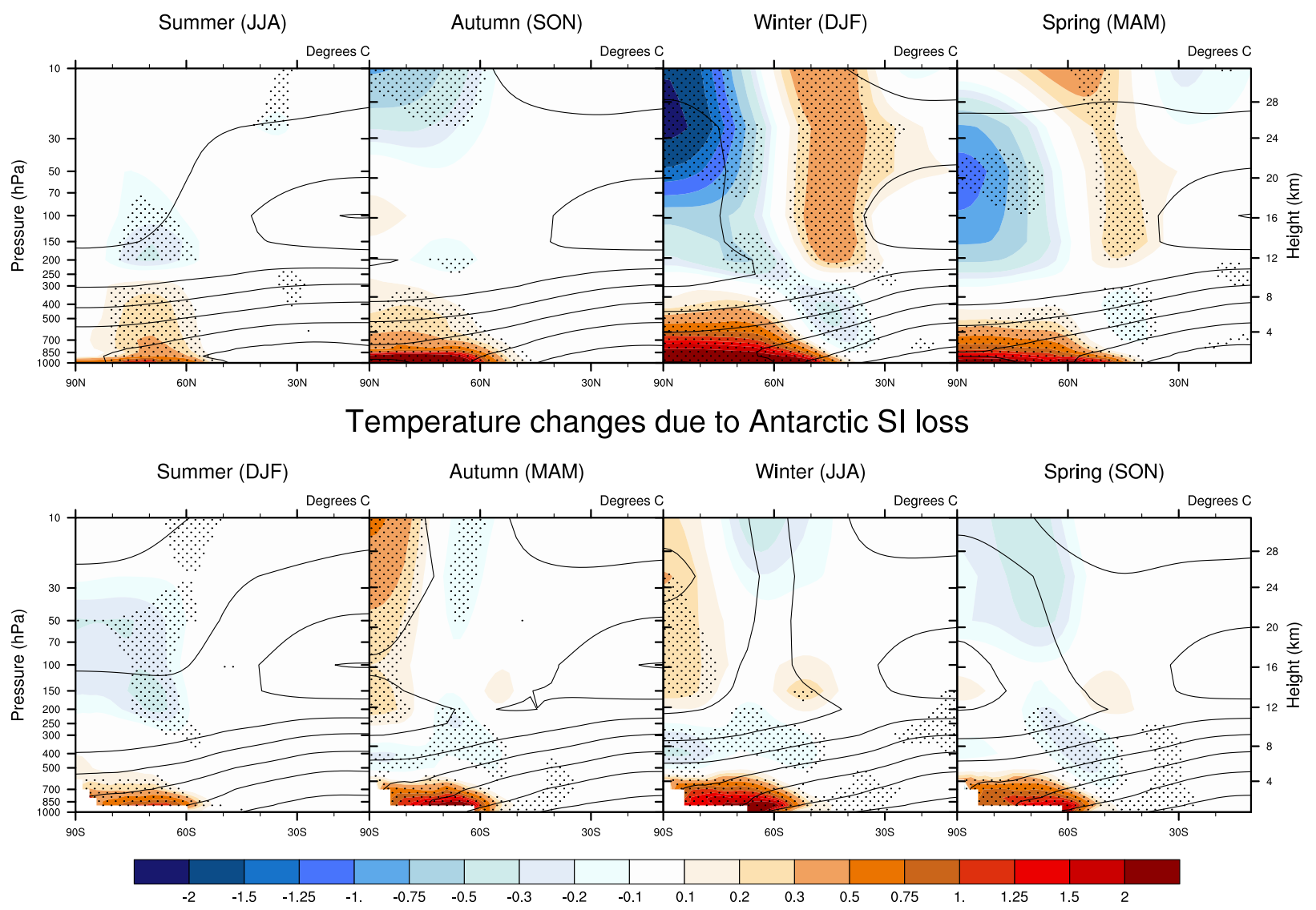

FIG. 3. Zonally averaged temperature changes throughout the atmosphere for the four seasons in response to Arctic and Antarctic future sea ice loss (shaded contours) on top of the control climatological temperature structure (black contours) with contour increments of $15^{\circ} \mathrm{C}$. Stippling denotes a statistically significant response at the $95 \%$ confidence level. Note that for ease of comparison, the latitudes for the Northern Hemisphere have been reversed. 


\section{Zonal wind change due to Arctic SI loss}

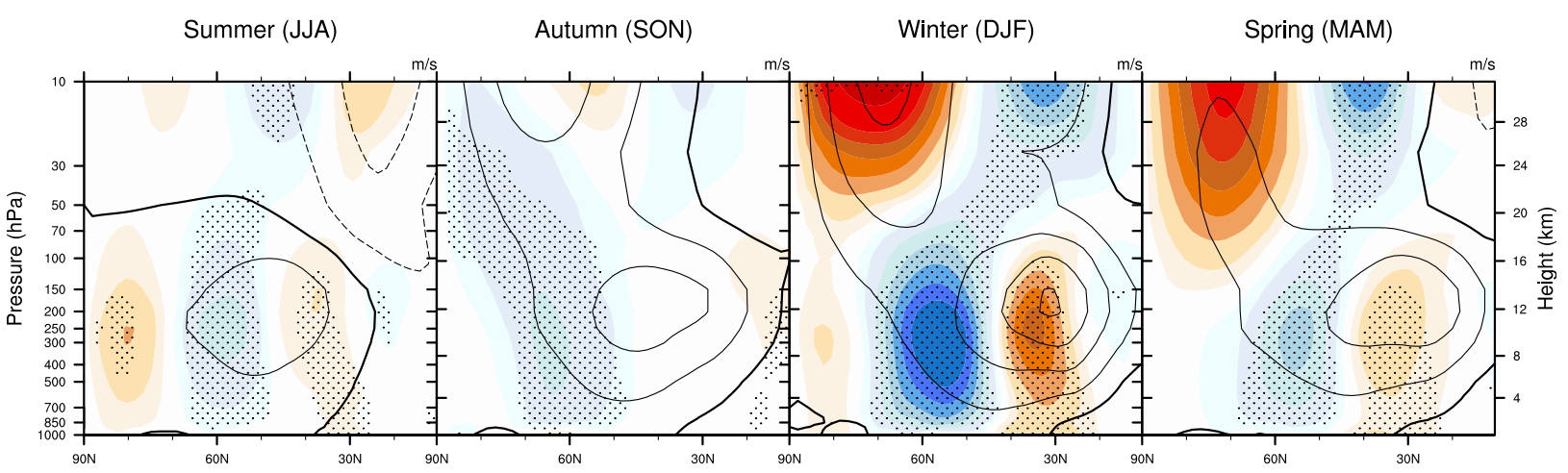

Zonal wind change due to Antarctic SI loss

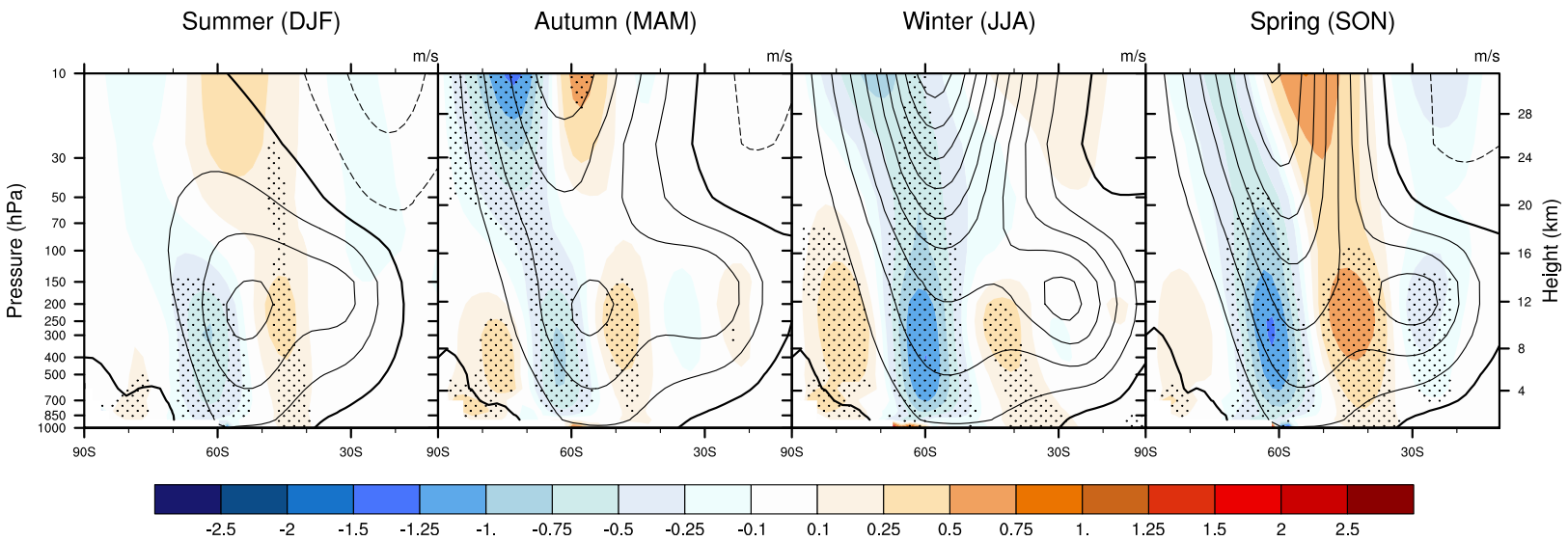

FIG. 4. As in Fig. 3, but for zonally averaged zonal wind. Contour increments of the control climatological zonal wind structure have increments of $10 \mathrm{~m} \mathrm{~s}^{-1}$ with the bold line being zero and the dashed lines indicating negative values.

future sea ice losses in the four seasons, overlaid on to the climatological temperature structure from the control experiment (contours). High levels of local warming are evident at high latitudes over the regions of sea ice loss. In both hemispheres, the response peaks in winter and is smallest in summer: this corresponds to the seasonal cycle of the net surface flux. It can be seen that, in the troposphere, the response in the Arctic shows larger seasonal variations whereas, in the Antarctic, the temperature response is more similar throughout the year. Examining the first harmonic of the area-averaged temperature response in the lower troposphere, the Arctic response has a seasonal cycle amplitude that is 2.1 times larger than that for the Antarctic response, and is significantly different at a $99 \%$ level.

The local warming is clearly confined, both latitudinally and vertically. In neither hemisphere we find a large mid- to upper-tropospheric warming, although the warming extends slightly higher in the Arctic (up to $400 \mathrm{hPa}$ ) compared to the Antarctic (up to $600 \mathrm{hPa}$ ).
Note also a small cooling response above $600 \mathrm{hPa}$ in the Antarctic for most of the year. The largest stratospheric temperature changes are found in the Arctic in winter, but the stratospheric response is examined in section $3 \mathrm{~b}$ below.

Figure 4 shows the zonally averaged zonal wind response throughout the whole atmosphere to projected Arctic and Antarctic sea ice loss (shaded). Again, it is clear that the tropospheric zonal wind response to Antarctic sea ice loss is less seasonally confined than for the Arctic case, although the biggest response occurs in spring. In the Arctic, the largest wind changes are seen in the stratosphere; however, even with our century-long integrations, most of the stratospheric response is not statistically significant because of the high variability in the Arctic stratosphere. In agreement with most previous studies, we find that melting sea ice causes an equatorward shift in the tropospheric jet in both hemispheres. This agrees with the recent finding of Baker et al. (2017) who suggest that, as long as it is located on 


\section{U 700hPa, Arctic SI loss}
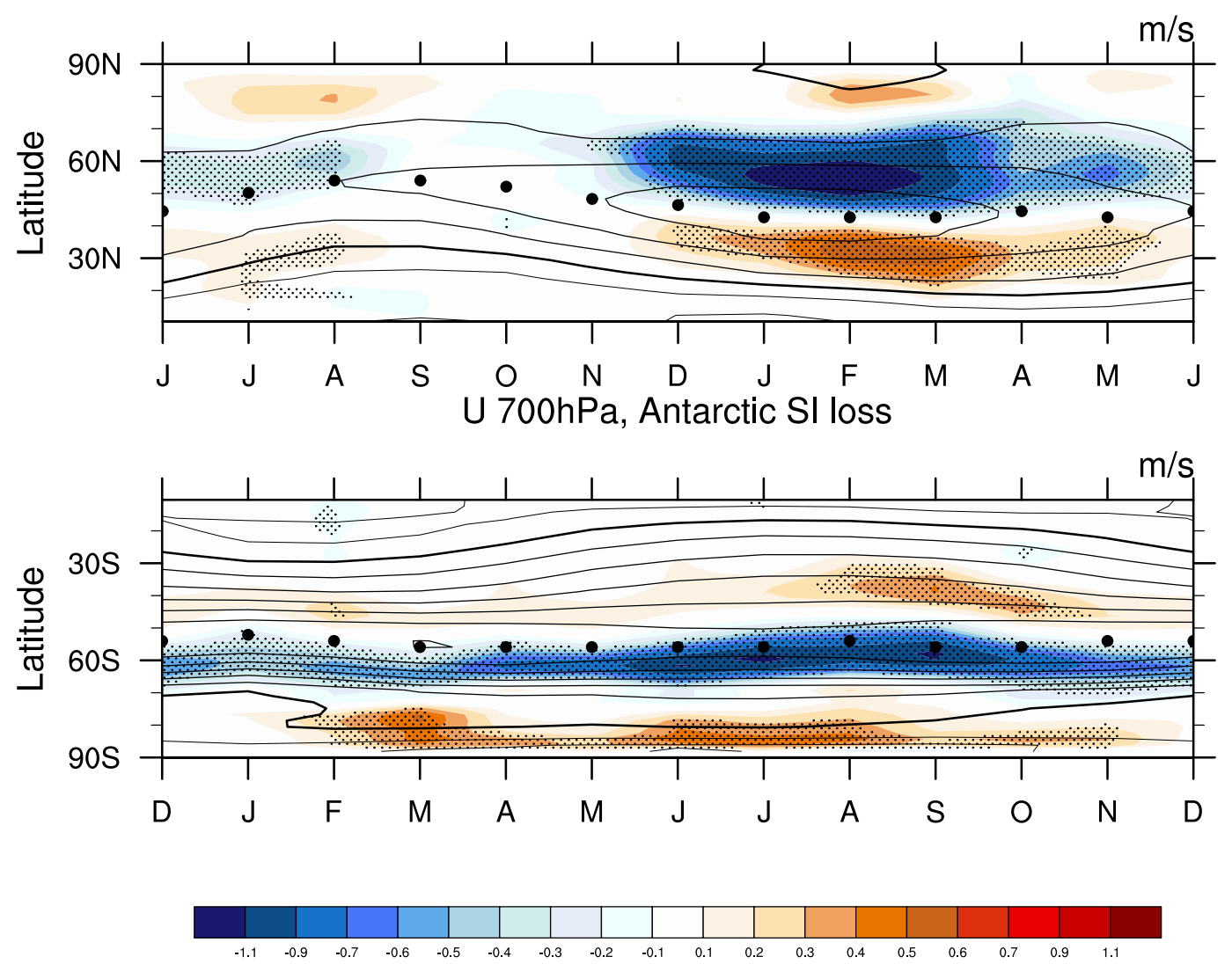

FIG. 5. Seasonal cycle of zonally averaged zonal wind changes at $700 \mathrm{hPa}$ in response to Arctic and Antarctic future sea ice loss (shaded contours) on top of the control climatological zonal wind structure (black contours). Contour increments of the control climatological zonal wind structure have increments of $3 \mathrm{~m} \mathrm{~s}^{-1}$ with the bold line being zero. Stippling denotes a statistically significant response at the $95 \%$ confidence level. The latitudinal location of the maximum zonal wind is shown with the black circles. Note that months have been shifted to align northern and austral seasons.

the jet's poleward flank, a local source of heating will move the tropospheric jet equatorward. The key point is that both Arctic and Antarctic projected sea ice losses would provide a negative feedback on the poleward tropospheric jet shift associated with increasing greenhouse gases in each hemisphere (Yin 2005; Lorenz and DeWeaver 2007; Barnes and Polvani 2013; Grise and Polvani 2014). Comparing to WACCM RCP8.5 simulations, according to these simulations sea ice losses would reduce the end of the century poleward winter jet shift by roughly $30 \%-40 \%$ [assuming the relationship remains linear; McCusker et al. (2017)].

The tropospheric zonal wind response to future sea ice loss throughout the year is illustrated in Fig. 5, which shows the entire seasonal cycle of zonal wind response at $700 \mathrm{hPa}$. This plot best conveys the difference in the seasonality of the response to future Arctic and Antarctic sea ice loss. The latitudinal position of the maximum zonal wind of the control run is indicated by the black dots, to demonstrate that both Arctic and Antarctic sea ice loss result in jet shifts, although as discussed later Antarctic sea ice loss also causes a weakening of the jet. These results disagree with the findings of Kidston et al. (2011) who find no apparent effect on the zonal wind. The major difference between the atmospheric responses in the two hemispheres is that Antarctic sea ice losses causes an equatorward shift of the eddy-driven jet throughout the entire calendar year, whereas the Arctic response is largest between December and May and not significant at all in the autumn. Last, we note that the peak response to future Antarctic sea ice loss is somewhat smaller than in the Arctic, albeit statistically significant for every month of the year.

To further elucidate the response of the tropospheric jet, the top panel of Fig. 6 compares the effect of Arctic and Antarctic sea ice on two important parameters 


\section{Jet shift response}

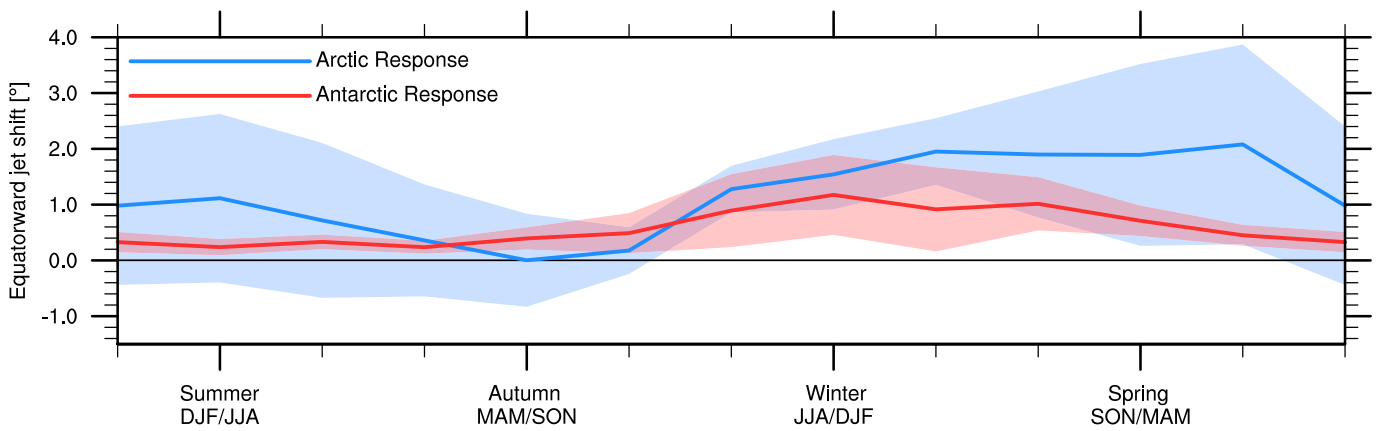

Jet strength response

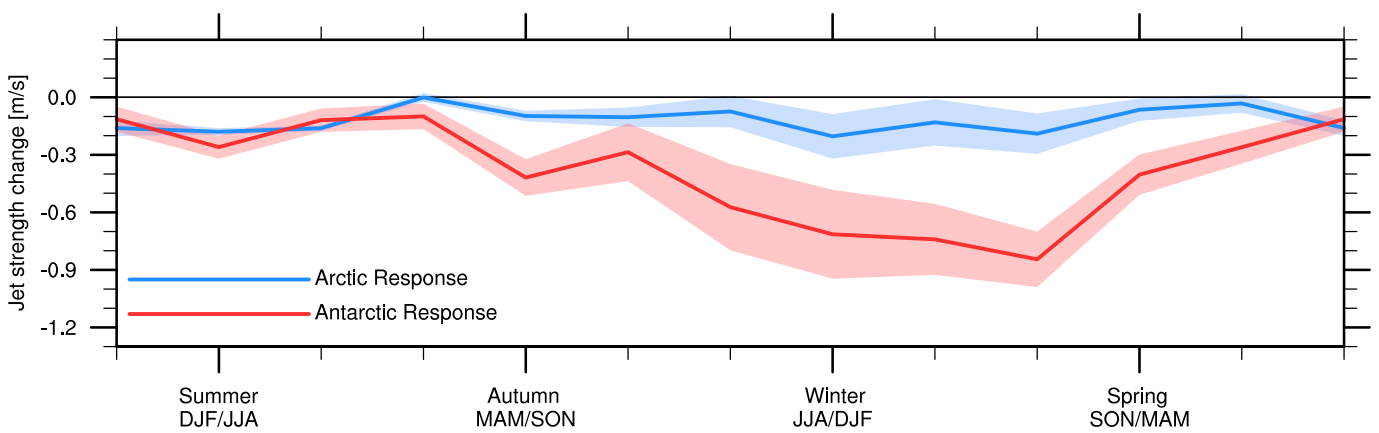

FIG. 6. Seasonal cycle of response of the Northern Hemisphere and Southern Hemisphere tropospheric jet (at $700 \mathrm{hPa}$ ) to sea ice loss. (top) The equatorward jet shift response and (bottom) the change in jet strength, with the negative values indicating a slowdown of the jet. For information on how these parameters were calculated, refer to section 2. Shading indicates the $+/-2 \sigma$ envelope. Note that months have been shifted to align northern and austral seasons.

describing the jet: the jet maximum speed and its latitudinal location (details about how these parameters are calculated are included in section 2). Consistent with Fig. 5, the response of the Southern Hemisphere tropospheric jet is a small but significant equatorward shift in every month, whereas the jet response to Arctic sea ice loss is not statistically significant for roughly a third of the year. The amplitude of the seasonal cycle of the response to Arctic sea ice loss is roughly double that of the Antarctic response, similar to the response of nearsurface temperature discussed previously.

Interestingly, one important feature where Antarctic sea ice loss seems to have a larger impact on than Arctic sea ice loss is the jet strength, shown in the bottom panel of Fig. 6. While the impact of sea ice loss is to weaken the tropospheric jet in both hemispheres, Antarctic sea ice loss has a significantly larger effect. This is consistent with Bracegirdle et al. (2018) who reported a stronger relationship between Antarctic sea ice extent and jet strength, than between sea ice extent and the latitudinal location of the jet. Although Bader et al. (2013) do not decompose the jet response into the strength and location, it seems that their results tell a similar story (although their experiment only examines the summertime). The role of jet strength could offer a partial explanation of why, even though the seasonal cycle of total forcing to the atmosphere is the same in both hemispheres, the response of the Southern Hemisphere jet latitude varies less seasonally than the Northern Hemisphere jet latitude; rather, these seasonal variations manifest themselves in a weakening of the jet.

Figure 7 shows the zonal wind response at $700 \mathrm{hPa}$ for each of the four seasons. The tropospheric zonal wind response due to future Antarctic sea ice loss is largely zonally symmetric, unlike the regional response to Arctic sea ice loss. One would have predicted a more zonal response in the Southern Hemisphere for two reasons: 1) the projected end of the century sea ice losses, seen in Fig. 1, are approximately zonally symmetric in the Antarctic and regionally confined in the Arctic; and 2) the effects are acting upon a climatological state that is far more zonally symmetric in the Southern Hemisphere (Kushner et al. 2001). In contrast, in response to melting Arctic sea ice, WACCM's primary center of action 


\section{Arctic 700hPa Zonal Wind Change}

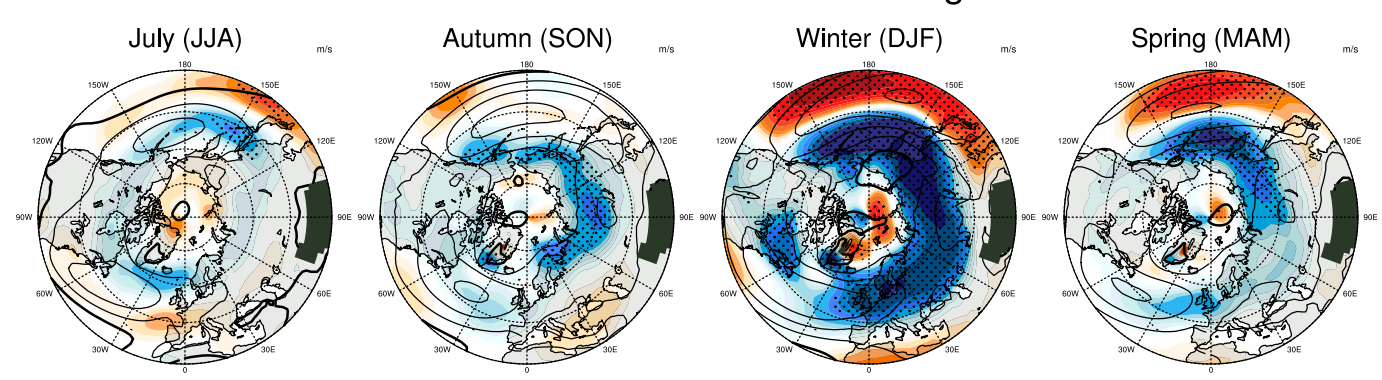

\section{Antarctic $700 \mathrm{hPa}$ Zonal Wind Change}
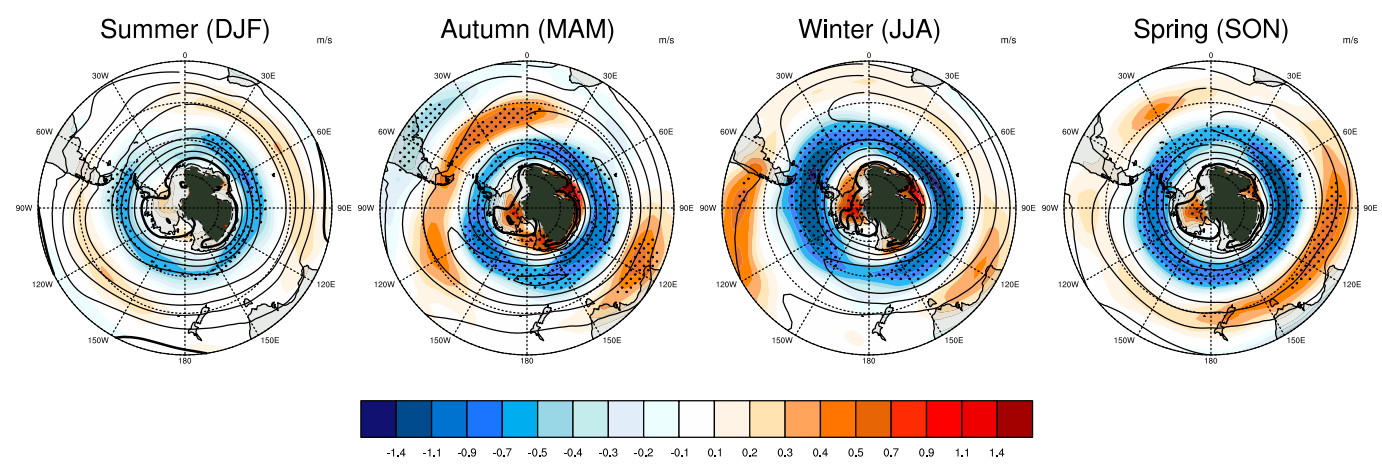

FIG. 7. 700-hPa zonal wind response to Arctic and Antarctic future sea ice loss (contours) on top of the control climatological 700-hPa zonal wind structure (black contours) with contour intervals of $4 \mathrm{~m} \mathrm{~s}^{-1}$. Stippling denotes a statistically significant response at the $95 \%$ confidence level. Some values are not plotted because of topography.

seems to be over the Pacific basin (not the North Atlantic): this is a common feature of many climate models (Gong et al. 2017).

\section{b. Stratospheric response}

Figure 8 shows the seasonal cycle of the stratospheric zonal wind response to future sea ice loss, as in Fig. 5, but at $10 \mathrm{hPa}$ rather than $500 \mathrm{hPa}$. One can easily see that while the tropospheric responses to Arctic and Antarctic sea ice loss are quite similar, the stratospheric responses are not. Arctic sea ice loss results in a strengthening of the polar vortex in our model between December and March, although the reader should note that much of it is not statistically significant. Our findings differ from many other modeling studies (Kim et al. 2014; Peings and Magnusdottir 2014), and perhaps most interestingly, S15, who used a very similar experimental design and the same model as we do. Although the stratospheric response in boreal spring is the same, S15 find a slowdown of the polar vortex in winter. We offer two possible explanations of this discrepancy: 1) given that the changes are small relative to the climatological conditions and much of the changes are not statistically significant, the differences could simply arise from the large levels of internal variability in the stratosphere; or 2) our study imposes slightly more sea ice loss in the Pacific sector (e.g., in the Sea of Okhotsk in winter) than S15 and, as a consequence, wave suppression could be more pronounced, leading to a stronger polar vortex. If the $150-\mathrm{yr}$ integration is broken down into three 50-yr chunks, 1 of those 3 time periods shows a large weakening of the winter stratospheric vortex in the Northern Hemisphere, rather than a strengthening (not shown). The fact that the sign of the response is not consistent throughout the integration suggests that internal variability might be the most important factor behind these differences.

The largest, and robust, stratospheric response to Antarctic sea ice loss, in Fig. 8, is seen in austral autumn (MAM): it consists of a weakening of the polar vortex on its poleward flank and a slight strengthening on its equatorward flank. Although these responses are statistically significant, their amplitudes are small and constitute only a few percent of the climatological state, with the Southern Hemisphere polar vortex being much stronger than its northern counterpart. It is possible that this small stratospheric response could be intensified when the atmospheric model is coupled to an interactive ocean (Smith et al. 2017), but that remains to be established for our model. 


\section{U 10hPa, Arctic SI loss}

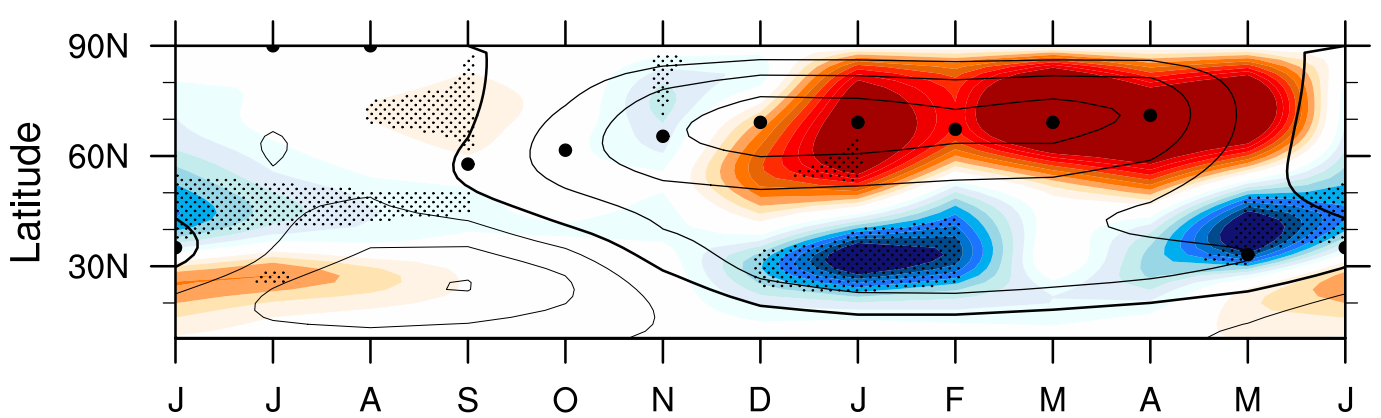

\section{$\mathrm{U}$ 10hPa, Antarctic SI loss}
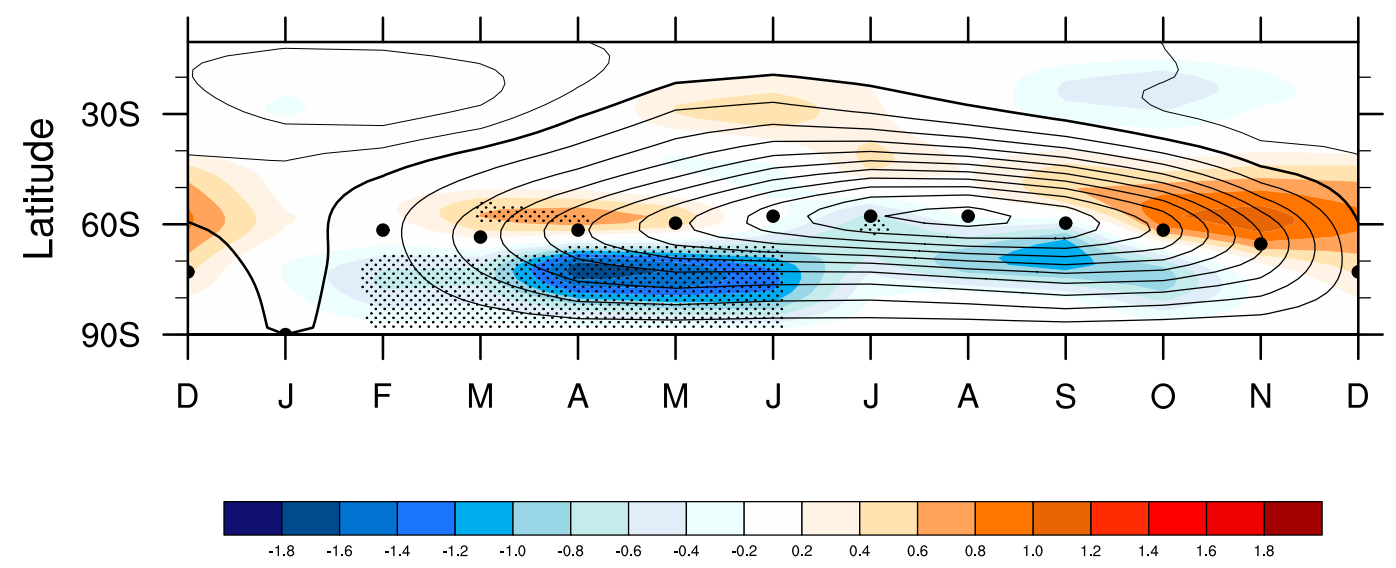

FIG. 8. As in Fig. 5, but for zonally averaged zonal wind changes at $10 \mathrm{hPa}$. Contour increments of the control climatological zonal wind structure have increments of $10 \mathrm{~m} \mathrm{~s}^{-1}$ with the bold line being zero.

To investigate the stratospheric responses further, Fig. 9 shows the stratospheric Eliassen-Palm (E-P) flux divergence response to Arctic and Antarctic sea ice loss. E-P flux divergence quantifies wave dissipation. Consistent with the stratospheric wind response, the largest change E-P flux divergence in the stratosphere is during boreal winter in response to Arctic sea ice loss. The increase in E-P flux divergence (reduced wave dissipation) is related to a suppression of upward wave propagation into the stratosphere (not shown), and thus a strengthened stratospheric jet. The E-P flux divergence response in the Antarctic, largest in austral spring and autumn, is weaker and does not impact the strength of the stratospheric jet. Projected sea ice losses in the Antarctic are highly zonally symmetric, and thus might not cause large changes in planetary waves and the stratospheric circulation.

Leveraging the fact that WACCM has interactive stratospheric chemistry, we now investigate the impact of sea ice loss on stratospheric ozone concentrations. Changes in stratospheric ozone have been shown to have an important impact upon the climate system
(Previdi and Polvani 2014). Analyzing the same set of runs as in S15, Sun et al. (2014) find that Arctic sea ice loss causes a significant reduction of total column ozone in the spring, by over 30 Dobson units. Figure 10 shows the response of total column ozone to Arctic and Antarctic sea ice loss in our runs. In agreement with Sun et al. (2014), we find a reduction of total column ozone in the spring (although of roughly half their magnitude), and this reduction extends back through the winter to November. This response is consistent with the stratospheric cooling seen in these seasons. The differences in the amplitude of stratospheric ozone seen in these two set of experiments might be linked to the different climatological conditions in the control runs; Sun et al. (2014) analyze a base period with reduced Arctic stratospheric ozone owing to the high levels of hydrocarbons in their simulations (Manney et al. 2011). These results further highlight how sensitive the stratospheric response is to the experimental design of these sea ice loss experiments.

In contrast to the reduction in stratospheric ozone seen in response to Arctic sea ice, Fig. 10 demonstrates 


\section{E-P Flux Div. 10hPa, Arctic SI loss}

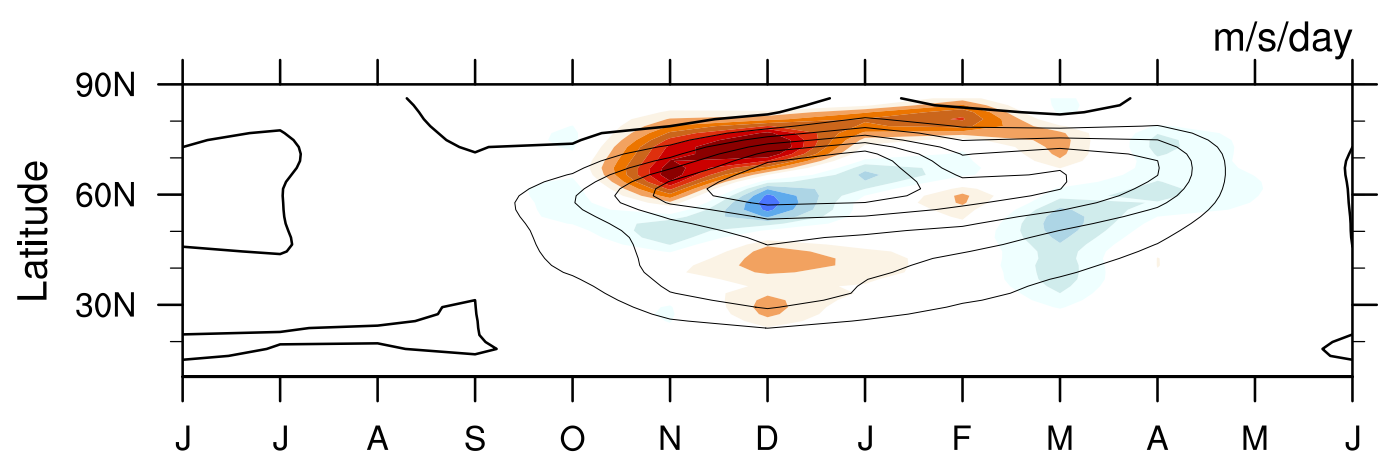

E-P Flux Div. 10hPa, Antarctic SI loss

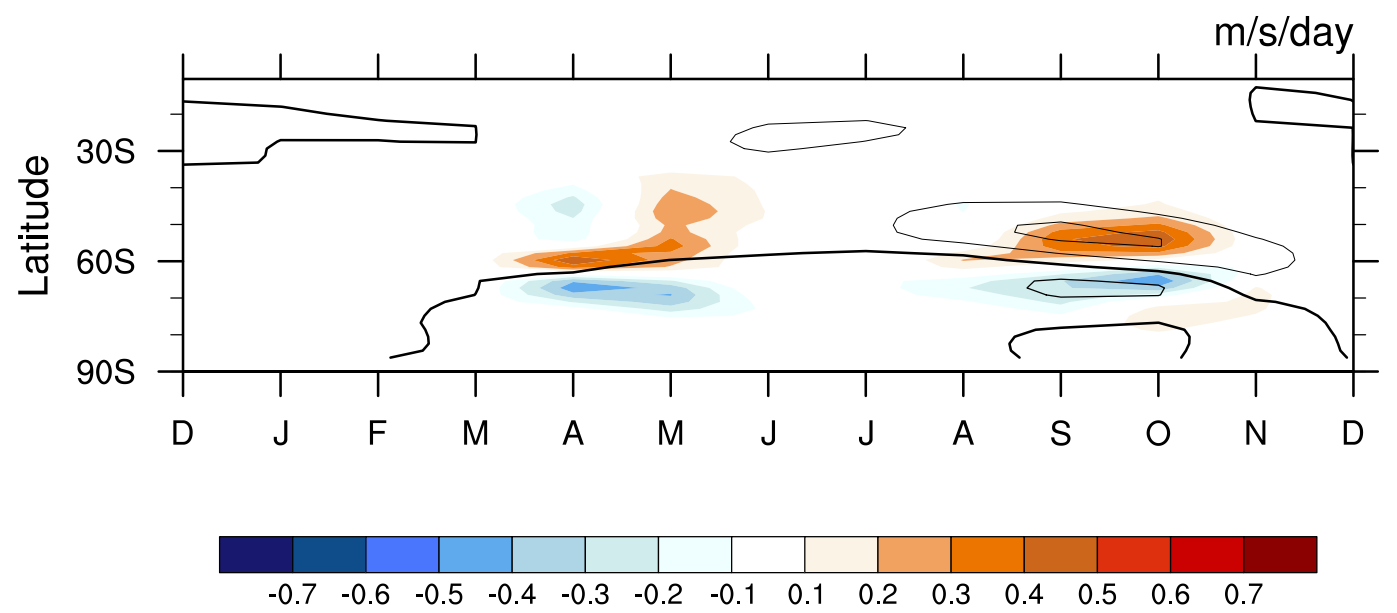

FIG. 9. Seasonal cycle of E-P flux divergence response (shaded contours) $\left(\mathrm{m} \mathrm{s}^{-1}\right.$ day $^{-1}$ ) at $10 \mathrm{hPa}$ in response to (top) Arctic and (bottom) Antarctic future sea ice loss overlayed on E-P flux divergence climatology (contours). Contour increments of the control climatological values have increments of $1 \mathrm{~m} \mathrm{~s}^{-1}$ day ${ }^{-1}$ with the bold line being zero and thin lines being negative. Only statistically significant responses have been shown. Negative values correspond to convergence and positive values correspond to divergence.

that Antarctic sea ice loss will actually cause an increase in stratospheric ozone, throughout much of the year. The response of ozone in the two hemispheres is consistent with the stratospheric temperature changes and their effect on ozone production rates. Another difference between the two hemispheres is that the response to Antarctic sea ice loss does not coincide with the season of maximum ozone depletion [SON; WMO (2014)]; austral spring actually exhibits the smallest response of any season. Note that in both hemispheres the response of stratospheric ozone concentrations to sea ice loss is small compared to the climatological values (especially in the Antarctic where the response constitutes only $1 \%-2 \%$ ). Long integrations are required to detect statistically significant differences [see Fig. 2b of Sun et al. (2014) for an indication of the large internal variability]. These changes in ozone are not considered large enough to influence the dynamics. Nevertheless, stratospheric ozone offers another instance with interesting differences between the atmospheric response to Antarctic and Arctic sea ice loss.

\section{c. Surface response}

Of potential societal and ecological importance, it is important to understand the surface response to Antarctic sea ice loss, starting from the surface temperature response that is shown in Fig. 11. First, note that the amplitude of warming in both hemispheres is similar with a maximum of roughly $10^{\circ} \mathrm{C}$ in the wintertime. Second, note that surface temperature changes in both hemispheres are broadly confined to the polar regions, with Arctic sea ice loss having a slightly larger effect toward the equator because the sea ice loss extends to lower latitudes. Third, our model shows that projected sea ice losses in the Southern Hemisphere have negligible impact upon the surface temperatures of the 


\section{Arctic Total Column Ozone}

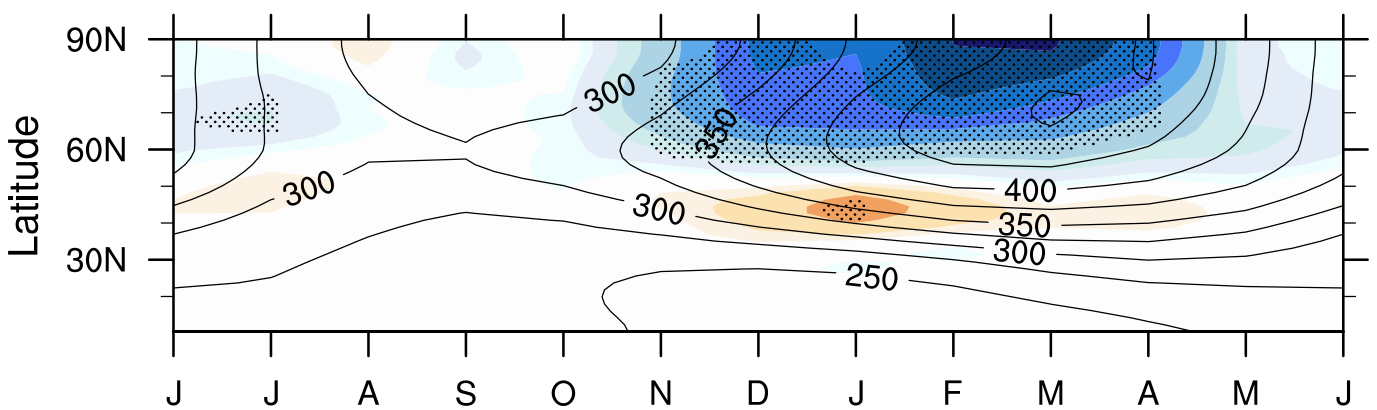

Antarctic Total Column Ozone
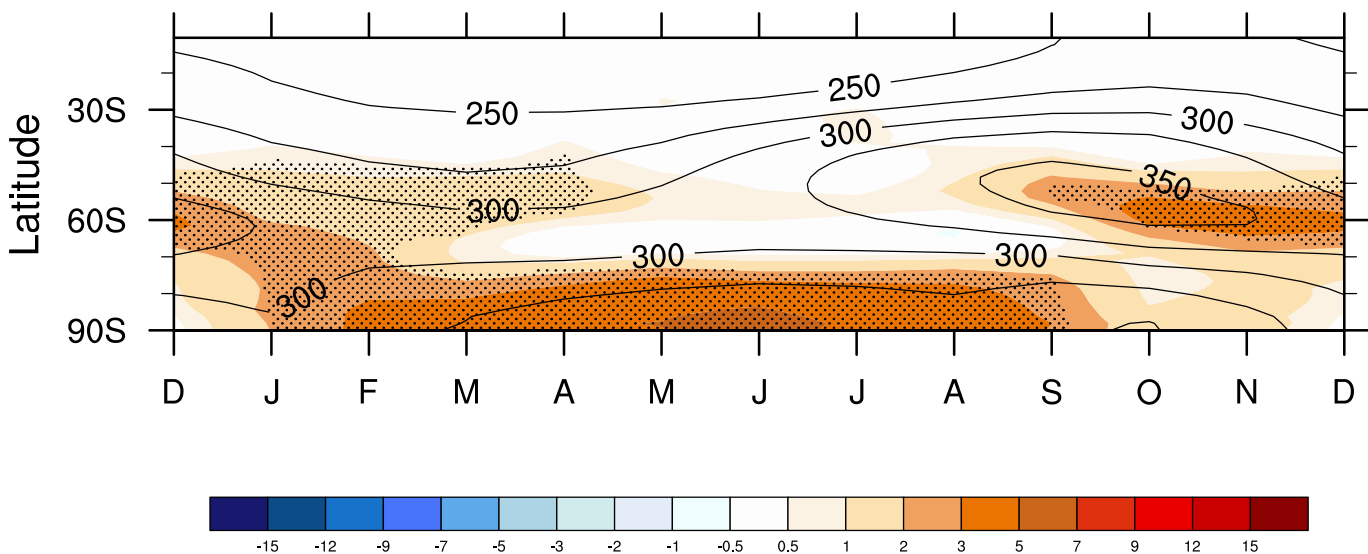

FIG. 10. As in Fig. 5, but for zonally averaged total column ozone (in Dobson units) in response to Arctic and Antarctic future sea ice loss. Contour increments of the control zonally averaged total column ozone structure have increments of 25DU. Stippling denotes a statistically significant response at the $95 \%$ confidence level.

interior of the Antarctic continent; however, we note a warming over the peninsula and the west Antarctic, which might have profound impacts upon the stability of Antarctic ice shelves (Trusel et al. 2015). It is probable that coupling to an interactive ocean would intensify the local warming further into the continent, and extend it in height (Smith et al. 2017), but we find it surprising how trapped near to the surface the response is in these runs, given the magnitude of the sea ice loss imposed. We also point out that Arctic sea ice loss causes a clear winter Eurasian cooling in these model integrations, between $1^{\circ}$ and $2^{\circ} \mathrm{C}$, in agreement with other studies (Cohen et al. 2014; Mori et al. 2014; S15; Wu and Smith 2016); however this response is not found by all studies (Screen et al. 2013; McCusker et al. 2016; Sun et al. 2016) and it may partly depend on the magnitude of the sea ice forcing.

We conclude by examining how precipitation responds to Arctic and Antarctic sea ice loss (Fig. 12). It can be seen that precipitation changes correspond to changes in the net heat flux, shown in Fig. 1. Areas in which the net heat flux is positive (where the ocean is releasing energy into the atmosphere) are collocated with an increase in precipitation. Conversely, areas in which the net heat flux is negative (where the atmosphere is losing energy to the ocean) experience a decrease in precipitation. This similarity between the net heat flux and local precipitation is in agreement with the findings of Deser et al. (2010), who show that the seasonal cycle of the averaged net heat flux and the integrated precipitation are completely in phase with one another. In the Southern Hemisphere, changes in precipitation at low latitudes, which could be affected by the midlatitude jet are not significant. There is no significant change in precipitation over populated landmasses in the Southern Hemisphere, such as Australia or South America, as the response is confined near the regions of sea ice loss. These results suggest a small impact of Antarctic sea ice loss on the midlatitudes in the Southern Hemisphere, either through changes in temperature or precipitation. Including ocean coupling, however, could alter this conclusion, because ocean 


\section{Arctic Surface Temperature Change}

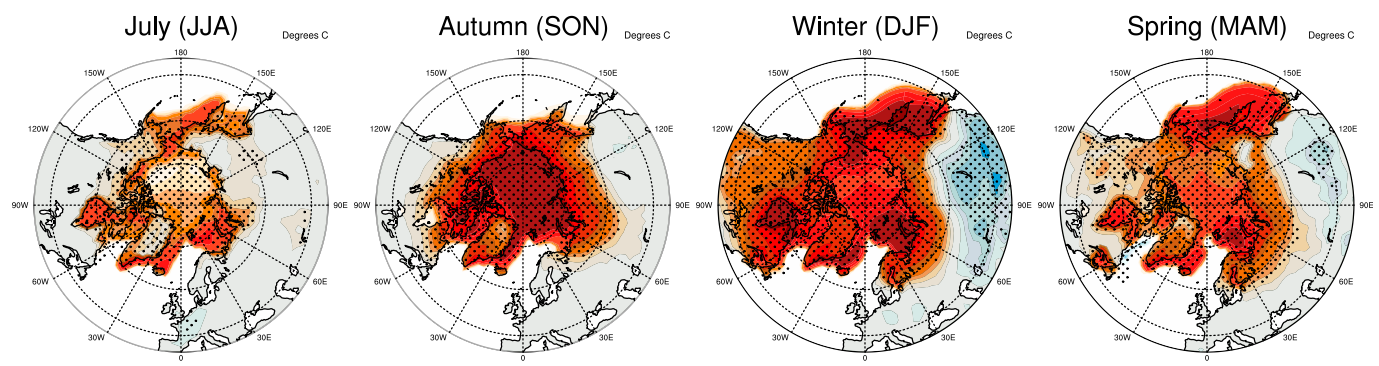

Antarctic Surface Temperature Change

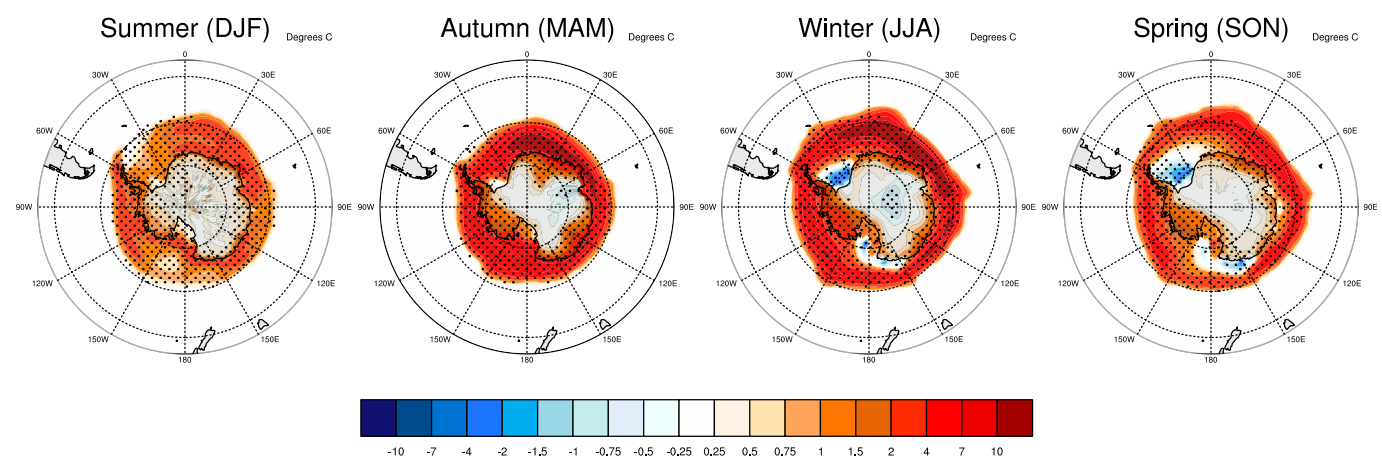

FIG. 11. Surface temperature response to Arctic and Antarctic future sea ice loss. Stippling denotes a statistically significant response at the $95 \%$ confidence level.

dynamics could act as a pathway to extend the impacts to the lower latitudes (Deser et al. 2016). Over the period 1979-2009, Smith et al. (2017) only find significant changes in precipitation near Australia or South America when the atmosphere is coupled to an interactive ocean.

\section{Summary and discussion}

\section{a. Summary}

We have performed a comprehensive set of model experiments to compare the atmospheric response to projected late twenty-first-century Arctic and Antarctic sea ice loss. There has been very little research on the effects of Antarctic sea ice loss, so our aim was to place the findings in the context of the Arctic, for which a vast literature exists. Our main conclusion is that future Antarctic sea ice loss is likely to have a significant impact on the atmospheric circulation throughout the year, with comparable magnitude to the impact of Arctic sea ice loss. We find that in both hemispheres, sea ice loss is associated with considerable surface and lowertropospheric warming in the polar regions; however, this localized warming is more confined in height and in latitude in the Antarctic case. Interestingly, the surface warming response in the Antarctic is unable to penetrate the interior of the Antarctic continent in these runs. Similarly, changes in precipitation are also confined to the polar regions. One possible reason for these two results, which motivates further study, is the lack of ocean coupling in these experiments. Sea ice loss in both hemispheres causes the tropospheric jet to shift equatorward; this is a negative feedback on the poleward jet shift associated with increased concentrations of greenhouse gases. The stratospheric response to Antarctic sea ice loss is smaller than the response to Arctic sea ice loss, and has a different seasonality. Comparison of our results with S15 suggests that stratospheric response to Arctic sea ice loss could be dominated by the large levels of internal variability in the stratosphere.

Perhaps the largest difference between the atmospheric effects of Arctic and Antarctic sea ice loss is their seasonal variability, even though the seasonal cycles of the net surface fluxes are similar. The peak response in the atmospheric circulation is seen in winter, but the amplitude of that response is significantly larger for Arctic sea ice loss than for Antarctic sea ice loss. However, the tropospheric response in the Antarctic is seen throughout the calendar year, whereas the response in the Arctic is mostly confined to winter and spring. The only impact of Antarctic sea ice loss that is of larger 


\section{Arctic Precip. Change}

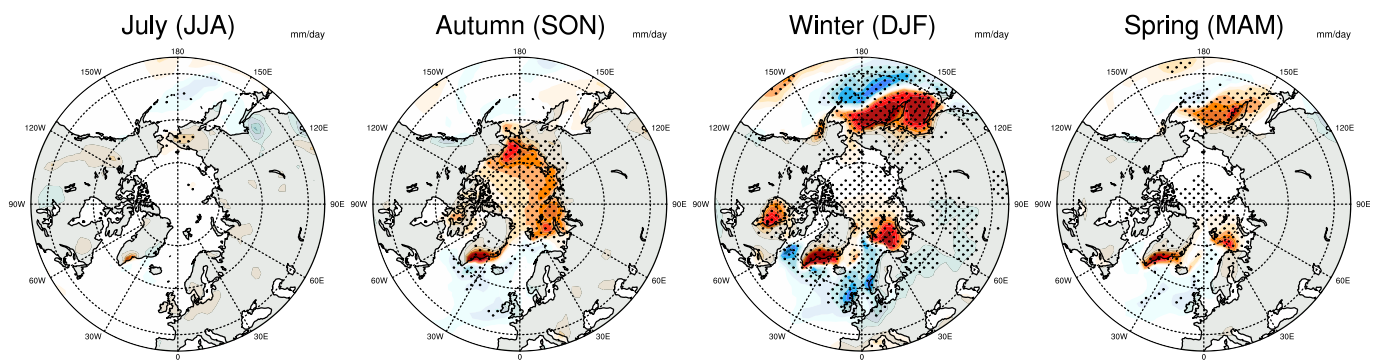

Antarctic Precip. Change

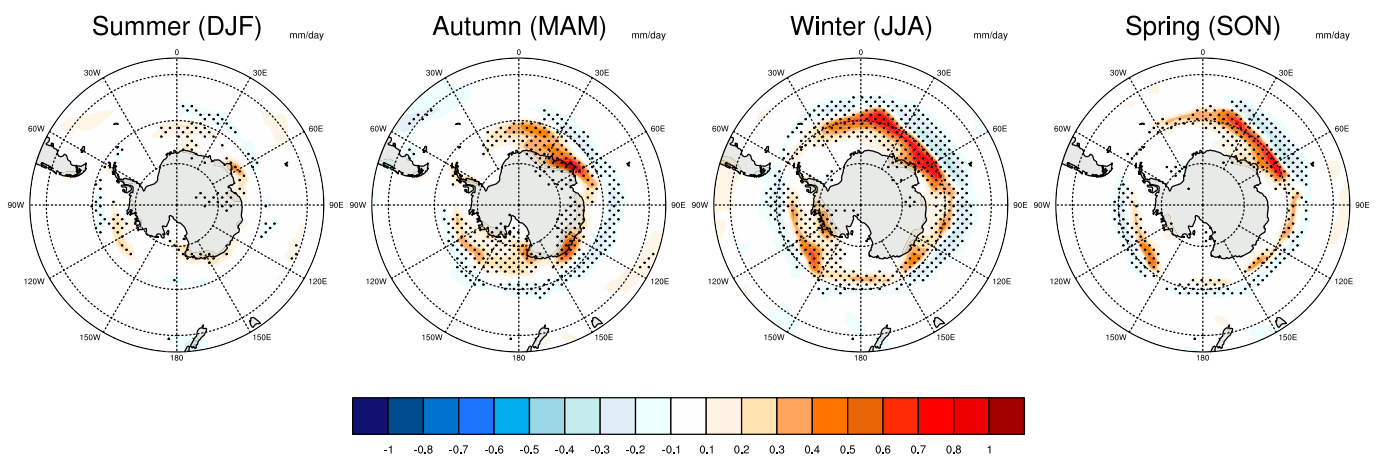

FIG. 12. Precipitation response to Arctic and Antarctic future sea ice loss $\left(\mathrm{mm} \mathrm{day}^{-1}\right)$. Stippling denotes a statistically significant response at the $95 \%$ confidence level.

amplitude than of its Arctic counterpart, is a weakening of the tropospheric jet, which peaks in winter. A key difference between two hemispheres is found in the zonality of the response to sea ice loss. For the Antarctic, the response of the atmospheric circulation is approximately zonally symmetric, whereas for the Arctic sea ice loss the response shows an important regional structure, with much of it located over the Pacific basin.

\section{b. Discussion}

One important caveat of these findings is that they are derived from a single climate model, so they are only as reliable as our model can be. It is obviously desirable to repeat our exercise with other models, to confirm these results and estimate the spread across models. One would want to understand why the results of Kidston et al. (2011) are in stark contrast to the findings of this study and that of Bader et al. (2013). Recent results from studies investigating the response to Arctic sea ice loss have highlighted the dependence of the results on the mean state of the atmosphere (Screen and Francis 2016; Osborne et al. 2017; Smith et al. 2017). Any biases in the background state of the control run, or the period chosen for the control run, could impact the results. This may explain some discrepancies between the results of this study and S15, even though the same model was used with a very similar experimental setup; also, the choice of 1955 rather than 2000 forcing could contribute to any differences. Further complicating the issue, there is evidence suggesting that the atmospheric response has a nonlinear relationship to the magnitude and location of the sea ice loss (Chen et al. 2016; Screen 2017b).

It is important to recall that recent observed sea ice trends in the Antarctic look very different to those simulated in WACCM, and in all other currentgeneration climate models; models show significant levels of high-latitude warming and a decline in sea ice extent in the last several decades. The literature is divided on whether the reason for this discrepancy is due to high levels of internal variability (Polvani and Smith 2013; Zunz et al. 2013; Gagne et al. 2015) or to, yet to be robustly identified, major flaws in the models (Marshall et al. 2014; Kostov et al. 2017; Rosenblum and Eisenman 2017). Whatever may be the case, it is hard to imagine that there will not be significant melting of Antarctic sea ice by the end of the century in response to increasing greenhouse gases, so our exercise is still informative. For instance, from our study we can learn something about possible circulation errors in the future projections. If, in the future, Antarctic sea ice loss occurs at a slower rate than projected by the current generation of climate 
models, then model projections are also likely to overestimate the future local warming and underestimate the poleward shift of the jet as well as the jet strength. Our results offer an initial estimate of the magnitude of these potential errors.

An important finding from our study is that, in our model, surface temperatures over the interior of the Antarctic continent appear not to respond to changes in sea ice conditions (even with the substantial end of the century sea ice loss imposed). In recent decades, the West Antarctic regions have been warming in the autumn (Schneider et al. 2012; Bromwich et al. 2013) but the eastern part of the continent has experienced a small cooling trend (Nicolas and Bromwich 2014). The extent to which these regional trends are due to internal variability (Smith and Polvani 2017) or are part of an anthropogenically forced response (Steig et al. 2009) is an open question. Our study suggests that cooling of the interior of Antarctica may be unrelated to changing sea ice conditions, but further investigations that couple an interactive ocean to the atmosphere are necessary to validate this hypothesis.

The next step to further our understanding of the effects of Antarctic sea ice loss, which we aim to complete soon, will be to repeat the same experiment using WACCM with a coupled ocean rather than fixed SSTs, using the approach of Deser et al. (2015). Literature on the Arctic suggests that including a coupled ocean can allow sea ice loss to have an impact on the low latitudes (Deser et al. 2016; Tomas et al. 2016; Blackport and Kushner 2017) and have an amplified effect on the high latitudes because of feedbacks (Deser et al. 2015; Smith et al. 2017). One would want to investigate whether this amplifying effect is as large in the Southern Hemisphere given the different configuration of oceans and landmasses in the Southern Hemisphere. In addition, it is important to understand how a coupled ocean might affect the difference in seasonality of the atmospheric response to Arctic and Antarctic sea ice loss that we have shown in this study.

Acknowledgments. The authors are grateful to three anonymous reviewers whose comments helped improve the manuscript. This work is funded by a Frontiers of Earth System Dynamics (FESD) grant from the U.S. National Science Foundation. The computations were carried out with high-performance computing support provided by NCAR's Computational and Information Systems Laboratory, which is sponsored by the National Science Foundation. The data produced for and analyzed in this paper are archived on the High Performance Storage System (HPSS) at the National Center for Atmospheric Research, and can be provided upon request.

\section{APPENDIX}

\section{Calculating Net Surface Heat Flux and Net Forcing to the Atmosphere}

The net surface heat flux is calculated as the sum of the longwave flux, the sensible heat flux, and the latent heat flux at the surface. We then average the net surface heat flux over all areas that have lost sea ice, the masks are calculated separately for each month. The area-average net surface heat flux has units of watts per square meter $\left(\mathrm{W} \mathrm{m}^{-2}\right)$. The net forcing to the atmosphere is calculated by multiplying the area-averaged heat flux by the total area of sea ice loss for each month. The net forcing to the atmosphere has units of $\mathrm{W}$.

\section{REFERENCES}

Bader, J., M. Flugge, N. Kvamsto, M. Mesquita, and A. Voigt, 2013: Atmospheric winter response to a projected future Antarctic sea-ice reduction: A dynamical analysis. Climate Dyn., 40, 2707-2718, https://doi.org/10.1007/s00382-012-1507-9.

Baker, H., T. Woollings, and C. Mbengue, 2017: Eddy-driven jet sensitivity to diabatic heating in an idealized GCM. J. Climate, 30, 6413-6431, https://doi.org/10.1175/JCLI-D-16-0864.1.

Barnes, E., and L. Polvani, 2013: Response of the midlatitude jets, and of their variability, to increased greenhouse gases in the CMIP5 models. J. Climate, 26, 7117-7135, https://doi.org/ 10.1175/JCLI-D-12-00536.1.

_ , and J. Screen, 2015: The impact of Arctic warming on the midlatitude jet-stream: Can it? Has it? Will it? Wiley Interdiscip. Rev.: Climate Change, 6, 277-286, https://doi.org/ 10.1002/wcc.337.

Blackport, R., and P. Kushner, 2017: Isolating the atmospheric circulation response to Arctic sea ice loss in the coupled climate system. J. Climate, 30, 2163-2185, https://doi.org/ 10.1175/JCLI-D-16-0257.1.

Bracegirdle, T., P. Hyder, and C. Holmes, 2018: CMIP5 diversity in southern westerly jet projections related to historical sea ice area: Strong link to strengthening and weak link to shift. J. Climate, $\mathbf{3 1}$, 195-211, https://doi.org/10.1175/JCLI-D-17-0320.1.

Bromwich, D., J. Nicolas, A. Monaghan, M. Lazzara, L. Keller, G. Weidner, and A. Wilson, 2013: Central West Antarctica among the most rapidly warming regions on earth. Nat. Geosci., 6, 139-145, https://doi.org/10.1038/ngeo1671.

Butler, A., D. Thompson, and R. Heikes, 2010: The steady-state atmospheric circulation to climate change-like thermal forcings in a simple general circulation model. J. Climate, 23, 3474 3496, https://doi.org/10.1175/2010JCLI3228.1.

Cai, D., M. Dameris, H. Garny, and T. Runde, 2012: Implications of all season arctic sea ice anomalies on the stratosphere. Atmos. Chem. Phys., 12, 11819-11831, https://doi.org/10.5194/ acp-12-11819-2012.

Chen, H., F. Zhang, and R. Alley, 2016: The robustness of midlatitude weather pattern changes due to Arctic sea ice loss. J. Climate, 29, 7831-7849, https://doi.org/10.1175/ JCLI-D-16-0167.1.

Cohen, J., and Coauthors, 2014: Recent Arctic amplification and extreme mid-latitude weather. Nat. Geosci., 7, 627-637, https://doi.org/10.1038/ngeo2234. 
Collins, M., and Coauthors, 2013: Long-term climate change: Projections, commitments and irreversibility. Climate Change 2013: The Physical Science Basis, T. F. Stocker et al., Eds., Cambridge University Press, 1029-1136.

Comiso, J., R. Gersten, L. Stock, J. Turner, G. Perez, and K. Cho, 2017: Positive trend in the Antarctic sea ice cover and associated changes in surface temperature. J. Climate, 30, 22512267, https://doi.org/10.1175/JCLI-D-16-0408.1.

Deser, C., R. Tomas, M. Alexander, and D. Lawrence, 2010: The seasonal atmospheric response to projected Arctic sea ice loss in the late twenty-first century. J. Climate, 23, 333-351, https:// doi.org/10.1175/2009JCLI3053.1.

,$- \ldots$, and L. Sun, 2015: The role of ocean-atmosphere coupling in the zonal-mean atmospheric response to Arctic sea ice loss. J. Climate, 28, 2168-2186, https://doi.org/10.1175/ JCLI-D-14-00325.1.

_ L. Sun, R. Tomas, and J. Screen, 2016: Does ocean coupling matter for the northern extratropical response to projected Arctic sea ice loss? Geophys. Res. Lett., 43, 2149-2157, https:// doi.org/10.1002/2016GL067792.

Eisenman, I., T. Schneider, D. Battisti, and C. Bitz, 2011: Consistent changes in the sea ice seasonal cycle in response to global warming. J. Climate, 24, 5325-5335, https://doi.org/10.1175/ 2011JCLI4051.1.

Gagne, M., N. Gillett, and J. Fyfe, 2015: Observed and simulated changes in Antarctic sea ice extent over the past 50 years. Geophys. Res. Lett., 42, 90-95, https://doi.org/10.1002/ 2014 GL062231.

Gong, H., L. Wang, W. Chen, X. Chen, and D. Nath, 2017: Biases of the wintertime Arctic Oscillation in CMIP5 models. Environ. Res. Lett., 12, 014001, https://doi.org/10.1088/1748-9326/12/1/ 014001.

Grise, K., and L. Polvani, 2014: The response of midlatitude jets to increased $\mathrm{CO}_{2}$ : Distinguishing the roles of sea surface temperature and direct radiative forcing. J. Geophys. Res., 41, 6863-6871, https://doi.org/10.1002/2014GL061638.

Kidston, J., A. Taschetto, D. Thompson, and M. England, 2011: The influence of Southern Hemisphere sea-ice extent on the latitude of the mid-latitude jet stream. Geophys. Res. Lett., 38, L15804, https://doi.org/10.1029/2011GL048056.

Kim, B., S. Son, S. Min, J. Jeong, S. Kim, X. Zhang, T. Shim, and J. Yoon, 2014: Weakening of the stratospheric polar vortex by Arctic sea-ice loss. Nat. Commun., 5, 4646, https://doi.org/ 10.1038/ncomms5646.

Kostov, Y., J. Marshall, U. Hausmann, K. Armour, D. Ferreira, and M. Holland, 2017: Fast and slow responses of Southern Ocean sea surface temperatures to SAM in coupled climate models. Climate Dyn., 48, 1595-1609, https://doi.org/10.1007/ s00382-016-3162-z.

Kushner, P., I. Held, and T. Delworth, 2001: Southern Hemisphere atmospheric circulation response to global warming. J. Climate, 14, 2238-2249, https://doi.org/10.1175/ 1520-0442(2001)014<0001:SHACRT>2.0.CO;2.

Lorenz, D., and E. DeWeaver, 2007: Tropopause height and zonal wind response to global warming in the IPCC scenario integrations. J. Geophys. Res., 112, D10119, https://doi.org/ 10.1029/2006JD008087.

Manney, G., and Coauthors, 2011: Unprecedented arctic ozone loss in 2011. Nature, 478, 469-475, https://doi.org/10.1038/nature10556.

Marsh, D., M. Mills, D. Kinnison, J. Lamarque, N. Calvo, and L. Polvani, 2013: Climate change from 1850 to 2005 simulated in CESM1(WACCM). J. Climate, 26, 7372-7391, https:// doi.org/10.1175/JCLI-D-12-00558.1.
Marshall, J., K. Armour, J. Scott, Y. Kostov, U. Hausmann, D. Ferreira, T. Shepherd, and C. Bitz, 2014: The ocean's role in polar climate change: Asymmetric Arctic and Antarctic responses to greenhouse gas and ozone forcing. Philos. Trans. Roy. Soc., 372, 2019, https://doi.org/10.1098/rsta.2013.0040.

McCusker, K., J. Fyfe, and M. Sigmond, 2016: Twenty five winters of unexpected Eurasian cooling unlikely due to Arctic sea ice loss. Nat. Geosci., 9, 838-843, https://doi.org/10.1038/ ngeo2820.

, P. Kushner, J. Fyfe, M. Sigmond, V. Kharin, and C. Bitz, 2017: Remarkable separability of circulation response to Arctic sea ice loss and greenhouse gas forcing. Geophys. Res. Lett., 44, 7955-7964, https://doi.org/10.1002/2017GL074327.

McKenna, C., T. Bracegirdle, E. Shuckburgh, P. Haynes, and M. Joshi, 2018: Arctic sea ice loss in different regions leads to contrasting Northern Hemisphere impacts. Geophys. Res. Lett., 45, 945-954, https://doi.org/10.1002/2017GL076433.

Menendez, C., V. Serafini, and H. LeTreut, 1999: The effect of sea ice on the transient atmospheric eddies of the Southern Hemisphere. Climate Dyn., 15, 659-671, https://doi.org/ 10.1007/s003820050308.

Mori, M., M. Watanabe, H. Shiogama, J. Inoue, and M. Kimoto, 2014: Robust Arctic sea ice influence on the frequent Eurasian cold winters in past decades. Nat. Geosci., 7, 869-873, https:// doi.org/10.1038/ngeo2277.

Nicolas, J., and D. Bromwich, 2014: New reconstruction of Antarctic near-surface temperatures: Multidecadal trends and reliability of global reanalyses. J. Climate, 27, 8070-8093, https://doi.org/10.1175/JCLI-D-13-00733.1.

NSIDC, 2018: Sea ice index. NSIDC, accessed 5 April 2018, https:// nsidc.org/data/seaice_index.

Osborne, J., J. Screen, and M. Collins, 2017: Ocean-atmosphere state dependence of the atmospheric response to Arctic sea ice loss. J. Climate, 30, 1537-1552, https://doi.org/10.1175/ JCLI-D-16-0531.1.

Overland, J., J. Francis, R. Hall, E. Hana, S. Kim, and T. Vihma, 2015: The melting Arctic and midlatitude weather patterns: Are they connected? J. Climate, 28, 7917-7932, https://doi.org/ 10.1175/JCLI-D-14-00822.1.

Peings, Y., and G. Magnusdottir, 2014: Response of the wintertime Northern Hemisphere atmospheric circulation to current and projected Arctic sea ice decline: A numerical study with CAM5. J. Climate, 27, 244-264, https://doi.org/10.1175/ JCLI-D-13-00272.1.

Polvani, L., and K. Smith, 2013: Can natural variability explain observed Antarctic sea ice trends? New modelling evidence from CMIP5. Geophys. Res. Lett., 40, 3195-3199, https:// doi.org/10.1002/grl.50578.

Previdi, M., and L. Polvani, 2014: Climate system response to stratospheric ozone depletion and recovery. Quart. J. Roy. Meteor. Soc., 140, 2401-2419, https://doi.org/10.1002/qj.2330.

Raphael, M., W. Hobbs, and I. Wainer, 2011: The effect of Antarctic sea ice on the Southern Hemisphere atmosphere during the southern summer. Climate Dyn., 36, 1403-1417, https:// doi.org/10.1007/s00382-010-0892-1.

Rosenblum, E., and I. Eisenman, 2017: Sea ice trends in climate models only accurate in runs with biased global warming. J. Climate, 30, 6265-6278, https://doi.org/10.1175/ JCLI-D-16-0455.1.

Schneider, D., C. Deser, and Y. Okumura, 2012: An assessment and interpretation of the observed warming of West Antarctica in the austral spring. Climate Dyn., 38, 323-347, https:// doi.org/10.1007/s00382-010-0985-x. 
Screen, J., 2017a: Far-flung effects of Arctic warming. Nat. Geosci., 10, 253-254, https://doi.org/10.1038/ngeo2924.

2017b: Simulated atmospheric response to regional and panArctic sea ice loss. J. Climate, 30, 3945-3962, https://doi.org/ 10.1175/JCLI-D-16-0197.1.

— , and I. Simmonds, 2010: The central role of diminishing sea ice in recent Arctic temperature amplification. Nature, 464, 1334 1337, https://doi.org/10.1038/nature09051.

—_, and J. Francis, 2016: Contribution of sea ice loss to Arctic amplification is regulated by Pacific Ocean decadal variability. Nat. Climate Change, 6, 856-860, https://doi.org/10.1038/ nclimate3011.

, I. Simmonds, C. Deser, and R. Tomas, 2013: The atmospheric response to three decades of observed Arctic sea ice loss. J. Climate, 26, 1230-1248, https://doi.org/10.1175/JCLI-D-12-00063.1.

- and Coauthors, 2018: Elucidating the effects of arctic sea ice loss on Northern Hemisphere climate. Nat. Geosci., 11, 155163, https://doi.org/10.1038/s41561-018-0059-y.

Smith, D., N. Dunstone, A. Scaife, E. Fiedler, D. Copsey, and S. Hardiman, 2017: Atmospheric response to Arctic and Antarctic sea ice: The importance of ocean-atmosphere coupling and the background state. J. Climate, 30, 4547-4565, https://doi.org/10.1175/JCLI-D-16-0564.1.

Smith, K., and L. Polvani, 2017: Spatial patterns of recent Antarctic surface temperature trends and the importance of natural variability: Lessons from multiple reconstructions and the CMIP5 models. Climate Dyn., 48, 2653-2670, https://doi.org/ 10.1007/s00382-016-3230-4.

Steig, E., D. Schneider, S. Rutherford, M. Mann, J. Comiso, and D. Shindell, 2009: Warming of the Antarctic ice-sheet surface since the 1957 International Geophysical Year. Nature, 457, 459-463, https://doi.org/10.1038/nature07669.

Sun, L., C. Deser, L. Polvani, and R. Tomas, 2014: Influence of projected Arctic sea ice loss on polar stratospheric ozone and circulation in spring. Environ. Res. Lett., 9, 084016, https:// doi.org/10.1088/1748-9326/9/8/084016.

_, _ and R. Tomas, 2015: Mechanisms of stratospheric and tropospheric circulation response to projected Arctic sea ice loss. J. Climate, 28, 7824-7845, https://doi.org/10.1175/JCLI-D-15-0169.1.
_ J. Perlwitz, and M. Hoerling, 2016: What caused the recent "warm Arctic, cold continents" trend pattern in winter temperatures? Geophys. Res. Lett., 43, 5345-5352, https://doi.org/ 10.1002/2016GL069024.

Tomas, R., C. Deser, and L. Sun, 2016: The role of ocean heat transport in the global climate response to projected Arctic sea ice loss. J. Climate, 29, 6841-6859, https://doi.org/10.1175/ JCLI-D-15-0651.1.

Trusel, L., K. Frey, S. Das, K. Karnauskas, P. Munneke, E. van Meijgaard, and M. van den Broeke, 2015: Divergent trajectories of Antarctic surface melt under two twenty first century climate scenarios. Nat. Geosci., 8, 927-932, https://doi.org/ 10.1038/ngeo2563.

Vaughan, D., and Coauthors, 2013: Observations: Cryosphere. Climate Change 2013: The Physical Science Basis, T. F. Stocker et al., Eds., Cambridge University Press, 317-382.

Vihma, T., 2014: Effects of Arctic sea ice decline on weather and climate: A review. Surv. Geophys., 35, 1175-1214, https:// doi.org/10.1007/s10712-014-9284-0.

WMO, 2014: Scientific assessment of ozone depletion: 2014. Tech. Rep. 55, WMO Global Ozone Research and Monitoring Project, 328 pp.

Wu, Y., and K. Smith, 2016: Reponse of Northern Hemisphere midlatitude circulation to Arctic amplification in a simple atmospheric general circulation model. J. Climate, 29, 20412058, https://doi.org/10.1175/JCLI-D-15-0602.1.

Yin, J., 2005: A consistent poleward shift of the storm tracks in simulations of 21st century climate. Geophys. Res. Lett., 32, L18701, https://doi.org/10.1029/2005GL023684.

Zhang, P., Y. Wu, and K. Smith, 2018: Prolonged effect of the stratospheric pathway in linking Barents-Kara Sea sea ice variability to the midlatitude circulation in a simplified model. Climate Dyn., 50, 527-539, https://doi.org/10.1007/ s00382-017-3624-y.

Zunz, V., H. Goosse, and F. Massonnet, 2013: How does internal variability influence the ability of CMIP5 models to reproduce the recent trend in Southern Ocean sea ice extent. Cryosphere, 7, 451-468, https://doi.org/10.5194/ tc-7-451-2013. 